\title{
Glycan Analysis by Ion Mobility-Mass Spectrometry and Gas-Phase Spectroscopy Christian Manz ${ }^{1,2}$, Kevin Pagel ${ }^{1,2}$
}

Addresses

${ }^{1}$ Institute of Chemistry and Biochemistry, Freie Universität Berlin, Takustr. 3, 14195 Berlin, Germany

2 Fritz Haber Institute of the Max Planck Society, Faradayweg 4-6, 14195 Berlin, Germany

Corresponding Author: Pagel, Kevin (kevin.pagel@fu-berlin.de)

\section{Abstract:}

Due to the existence of numerous isomers, the in-depth analysis of glycans represents a major challenge. Currently, the majority of glycans are analysed using mass spectrometry (MS)-based techniques, which can provide information on regioisomers but usually fail to differentiate stereoisomers. A promising approach to overcome this limitation is to implement ion mobility spectrometry (IMS) as an additional gas-phase separation dimension. This review highlights recent developments in which IMMS was used as a tool for comprehensive glycan analysis or as rapid screening method for glycan feature analysis. Furthermore, we summarize a series of very recent investigations in which gas-phase spectroscopy is applied to study glycans and discuss the potential of the hyphenation between IM-MS and infrared (IR) spectroscopy as a future tool for glycomics and glycoproteomics.

\section{Introduction}

Glycans have long been known for their major role in the metabolism and structural properties of biological systems, especially for sensing in inter- and intramolecular interactions such as innate and adaptive immune responses [1]. Their underlying structure dictates their function and therefore comprehensive structural characterization of glycans is prerequisite to the elucidation of in vivo operation. However, in contrast to DNA and proteins, glycans often exhibit non-linear, branched, and stereochemically complex structures [2]. As a result, they possess a unique complexity, which is based on the type of building blocks (composition), the regiochemistry and branching (connectivity), and the stereochemistry (configuration) (Figure 1).

\section{a) Structural Formula of FLNH}

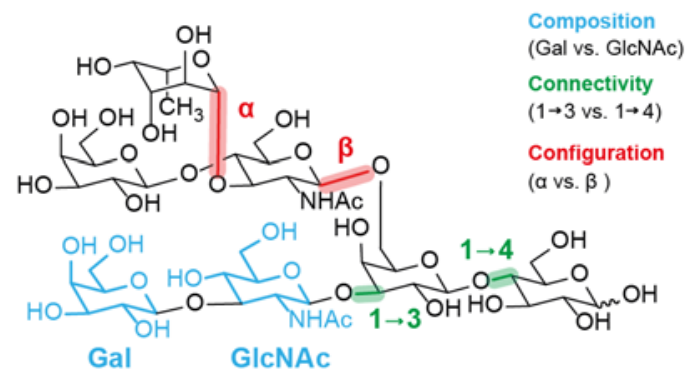

b) Symbol Nomenclature for Glycans

c) Symbol Nomenclature of $\mathrm{FLNH}$

$\begin{array}{ll}\text { Composition } & \begin{array}{l}\mathrm{E}=\mathrm{GlcNAc} \\ \mathrm{O}=\mathrm{Gal}\end{array} \\ \text { Connectivity } & \\ \text { Configuration } & -\beta \ldots \alpha \\ & \sim \text { unknown }\end{array}$

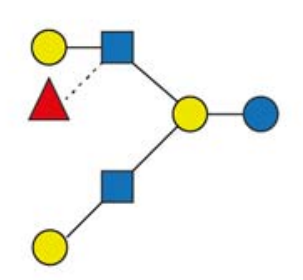

Figure 1: Visual representation of glycans. a) The most accurate depiction of glycans is the structural formula as shown for the milk oligosaccharide fucosyllacto-N-hexaose (FLNH). However, structural features such as the composition (blue), connectivity (green) and configuration (red) are often 
difficult to identify, due to the complex nature of oligosaccharides. b) The symbol nomenclature for glycans (SNFG) was introduced to allow a better visualisation of complex structures. Here, monosaccharides are illustrated using specific symbols, while the regio- and stereochemistry of the glycosidic bond is represented by the angle and the type of the connecting lines, respectively [3]. c) Depicting the milk oligosaccharide FLNH using the SNFG simplifies its visualisation and allows an easy identification of minute structural differences.

The combination of these structural categories frequently results in closely related structural isomers, which are often difficult to distinguish using established analytical tools. Furthermore, the analysis is complicated by the dynamic nature of monosaccharides, which can occur in multiple conformations [1]. They can undergo a process called mutarotation, which represents the structural rearrangement between $\alpha$ and $\beta$ configuration with an open chain form as intermediate, leading to an equilibrium between $\alpha$ and $\beta$ configuration. During this process a monosaccharide can also convert its more stable six-membered ring (pyranose) into the less preferred five-membered ring form (furanose).[4] In oligosaccharides, usually pyranoses are present and the flexibility is only retained for the building block at the reducing end. All these aspects make glycans a challenging target and it usually requires more than one isolated analysing tool for structural elucidation. Today, a variety of hyphenated techniques are applied to elucidate glycan structures, with the majority deriving directly from technologies developed in genomics and proteomics. The most prominent example is the combination of liquid chromatography (LC) and mass spectrometry (MS). This combination has proved to be useful for the separation of released glycans and the characterization of glycan composition [5]. However, carbohydrates are inherently very polar molecules that are difficult to be retained using stationary phases employed traditionally in reversed-phase chromatography. Therefore other stationary phases such as porous graphitized carbon (PGC) [6] or techniques such as hydrophilic interaction chromatography (HILIC) [7] need to be employed. Unfortunately, they often fail in the separation of glycoconjugates such as glycopeptides or glycolipids due to their mixed polarity. In addition, carbohydrates possess no strong, naturally occurring fluorophores, which hinders spectroscopic detection [8]. That is the reason why established LC-MS workflows in glycan analysis often involve a derivatization step to label the glycans with a fluorescent marker, which improves glycan identification but is time consuming and expensive. On the other hand, MS-based approaches struggle to distinguish between stereochemical information due to the presence of a variety of isomers with identical atomic composition and mass [9-12]. Sophisticated $\mathrm{MS}^{n}$ experiments can help to overcome this limitation, but they are elaborate and therefore of limited use in routine analysis [13]. Recently, other gas-phase analysis techniques such as ion mobility spectrometry (IMS) and gas-phase spectroscopy have emerged as promising alternatives. Here we summarize recent developments on the gas-phase structural analysis of oligosaccharides and glycopeptides.

\section{Ion Mobility Spectrometry}

IMS is a gas-phase separation technique in which ions are guided through a gas-filled drift cell by an electric field. On their way, they undergo collisions with a buffer gas, which separates them based on their charge, size and shape [14]. Coupling IMS with MS allows for the detection of the mobility-separated ions and the collection of mass-to-charge $(\mathrm{m} / \mathrm{z})$ and drift time information within one measurement. The obtained instrument-dependent drift time can furthermore be converted into a rotationally-averaged collision cross-section (CCS), which is correlated to the shape of an ion and can be used as additional identification parameter.

\section{Group Separation via IM-MS}

One of the main advantages of ion mobility mass spectrometry (IM-MS) is its universal separation power. Unlike LC separations, which are very sensitive to the stationary phase, IM-MS depends on the much more universal interaction principles of the investigated ions with the drift gas. Due to their distinct atomic composition, different molecular classes can exhibit a different mobility behaviour, especially when a polarizable gas such as nitrogen is used. This property can be used to significantly reduce the spectral complexity of acquired data [15]. It was recently demonstrated that whole molecular classes can be easily distinguished based on their trend lines in a plot of CCSs against $\mathrm{m} / \mathrm{z}$. For example, carbohydrates exhibit on average shorter drift times compared to lipids and peptides of the same $\mathrm{m} / \mathrm{z}$, allowing a rapid identification of each class [16]. This group separation can also be observed for different charge states of molecules (Figure 2) [17]. 

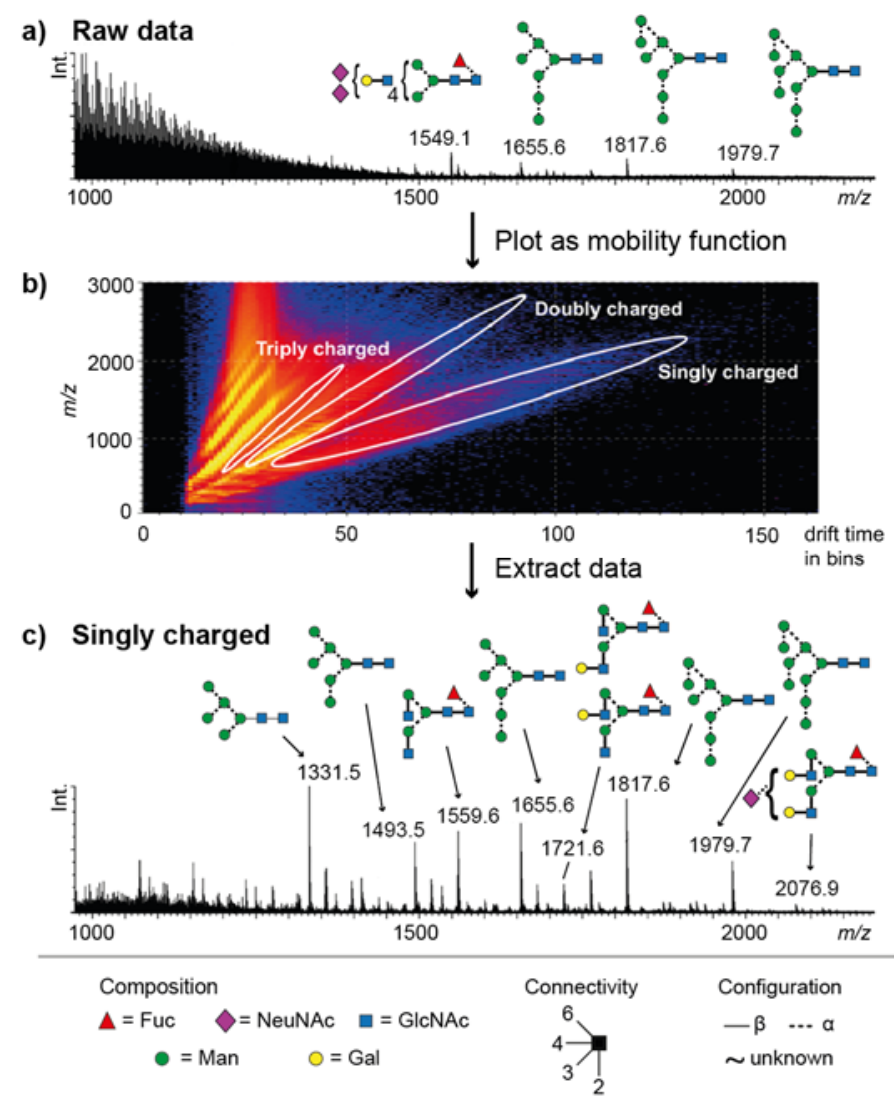

Figure 2: IM-MS analysis of glycans released from HIV-1 envelope glycoprotein gp120. The visual representation of the carbohydrates follows the symbol nomenclature for glycans (SNFG) [3]. a) Raw negative-ion ESI spectrum from N-glycans obtained from the release of gp120. b) DriftScope plot ( $\mathrm{m} / \mathrm{z}$ as function of drift time) of the raw data. The highlighted regions mark individual charge states of the released $\mathrm{N}$-glycans. $\mathrm{c}$ ) Spectrum of singly charged N-glycans extracted from the lower region of the DriftScope plot. (Adapted by permission from American Society for Microbiology: Journal of Virology [17], copyright 2015.)

Multiply charged ions generally exhibit a higher mobility than singly charged ions with the same $\mathrm{m} / \mathrm{z}$, resulting in individual signal groups (Figure $2 b$ ). Selectively extracted data from complex spectra enables significantly reduced signal-to-noise ratios and thus allows for improved molecular identification of signals (Figure 2c). HARVEY ET AL. used this approach to extract N-glycan profiles from crude incubation mixtures without further chemical clean-up [18]. It enabled the differentiation and analysis of distinct species within one measurement, therefore significantly decreasing the sample consumption. However, IM-MS is not limited to a coarse separation of molecular classes but can also be used to distinguish minor structural differences between isomers.

\section{Top-Down Fragment Analysis}

Recently, it was demonstrated that IM-MS can be used to distinguish small glycans as well as various glycopeptide isomers [19]. Fundamental work on the capabilities of IM-MS for glycan separation was done by HOFMANN ET AL., who investigated a set of six isomeric trisaccharides, which differed in their composition, connectivity, or configuration [20*0. A comparison of the CCSs of the intact deprotonated trisaccharides showed significant differences for configurational isomers (alpha vs. beta glycosidic bonds) as well as for connectivity isomers (1,4 vs. 1,3 glycosidic linkages), whereas compositional isomers could not be distinguished. The study revealed the fundamental ability of IM-MS to separate many glycan isomers in the gas phase. The results allow the conclusion that IM-MS-based methods have the potential to serve as powerful analysing tool for glycans. Essential for structural identification is the combination of mass and mobility information within a multidimensional dataset and the comparison of obtained data with known, authentic standards. Unlike retention times in LC, $\mathrm{m} / \mathrm{z}$ and CCSs are highly reproducible values when measured 
under controlled conditions. However, the assignment of drift peaks/CCSs to specific structural motifs can be complex due to the possibility of multiple ion conformations. In some cases, conformers can increase the number of drift peaks although the number of individual analytes remains the same, which complicates the analysis of the measured data [21]. The identification confidence can be increased by comparing obtained CCSs with reference values of comprehensive databases [22-24] or theoretical values. CCSs can be calculated theoretically by various methods [25-27], however, finding structural candidates for theoretical calculations is not an easy task due to various effects such as solvation interaction, charge localisation and especially due to the enormous structural flexibility of glycans as briefly described in the introduction.

The separation capability of IM-MS is mainly dependent on the resolution of the used instrument, which is often defined by the length of the applied IMS cell [28]. Increasing resolution therefore requires instrumental modification, which often is not feasible. Alternatively, IM separation can often be improved when adding sodium or other metal ions to an analyte. It is known that metal adducts can influence the conformation of carbohydrate ions [29]. Experiments investigating oligosaccharides adducted with group I metal ions showed a general trend of increasing drift times of adducted species proportional to the size of the metal ion. However, many metal adducts showed an isomer-specific drift time dependency, demonstrating the potential utility of metal/ carbohydrate interaction for improving the separation capability of glycans in IM-MS [30,31•*].

\section{a) Top-Down Fragment Analysis}

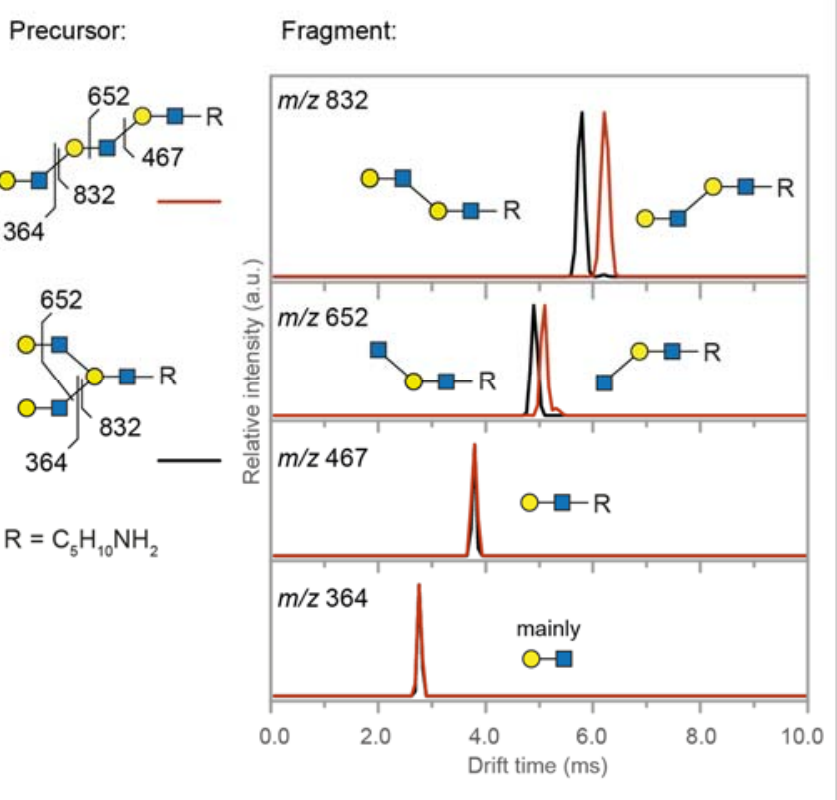

\section{b) Rapid Screening Technique}

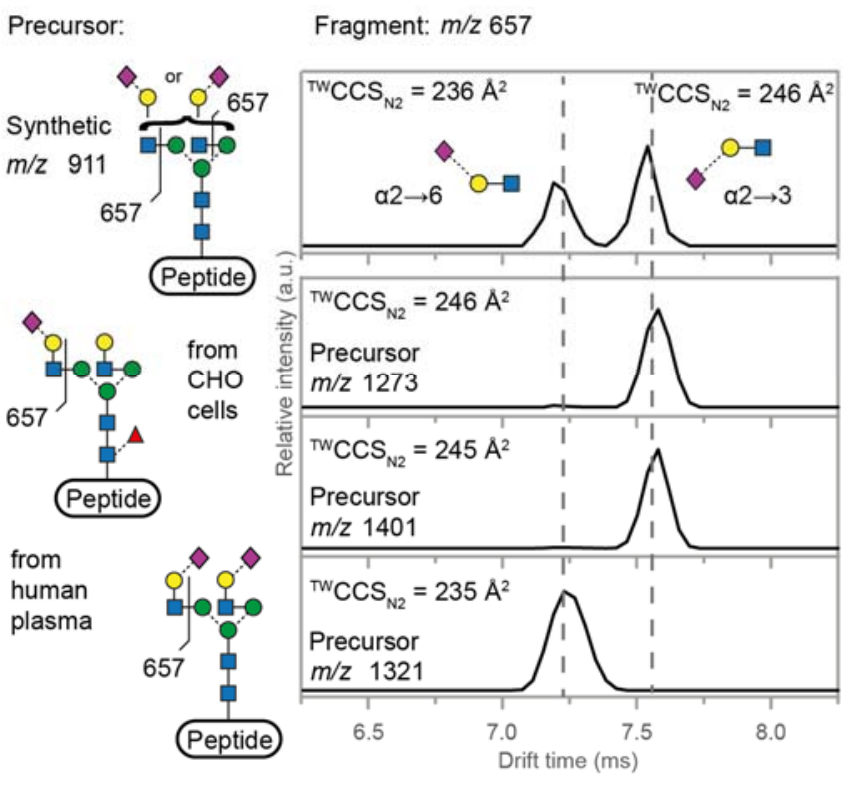

Figure 3: Application of IM-MS for structural analysis of complex carbohydrates. a) Top-down fragment analysis of two isomeric hexasaccharides. The fragmentation pattern is indicated for the two precursor structures and shown as respective fragment ATDs (Adapted by permission from Nature Publishing Group: Nature [20*0], copyright 2015.). b) The fragmentation of glycopeptide precursors leads to the generation of characteristic trisaccharide fragments $(\mathrm{m} / \mathrm{z} 657$ ), which can be used to rapidly identify the sialylation pattern (upper panel). This is shown exemplarily for tryptic digests of a1-antitrypsin generated in Chinese hamster ovary (CHO) cell lines or extracted from human plasma (lower panels). (Adapted with permission from Ref. [37] - Published by The Royal Society of Chemistry.)

Due to the decreasing impact of small structural differences on the overall shape, IM-MS separation becomes less efficient for larger glycans. This often complicates the differentiation of large intact ions. However, the fragmentation of intact precursors can lead to diagnostic fragments with distinct CCSs, from which information on the structure of the precursor can be deduced [32]. Collision-induced dissociation (CID) experiments on the Man3-GIcNAc2 pentasaccharide core of $\mathrm{N}$-glycans and fragments thereof showed the reproducibility of this approach [33]. Regardless of the nature of the precursor, similar arrival time distributions (ATDs) and CCSs were obtained for mono- and disaccharide fragments of similar structure [33]. One application of this approach was shown for the differentiation of isomeric Lewis and blood group epitopes. [34] Fragment trisaccharides of larger glycans showed 
characteristic ATDs when compared to drift times of intact trisaccharides and enabled the assignment of the underlying fucosylation pattern. This procedure can also be used as top-down fragment analysis, as demonstrated by HOFMANN ET AL (Figure 3) [20*]. Two isomeric hexasaccharides, which either exhibited a branched or a linear alignment of identical building blocks, were investigated (Figure 3a, precursor structures). As intact precursors, both oligosaccharides showed identical ATDs and CCSs. From the resulting di- to pentasaccharide fragments, however, it was possible to obtain detailed information on the structure and branching of the precursor ion (Figure 3a, fragment spectra of $n=2-4$ fragments). The analysis of fragment CCSs can therefore be more informative than analysing intact precursor ions [35] and has great potential to be used for the quality control and sequencing of larger, more complex glycans.

\section{Feature Analysis of Specific Glycan Motifs}

Fragment CCSs cannot only be used to piece together the structure of larger oligosaccharides, but also have great potential to be used for the rapid screening of characteristic features in glycans and other glycoconjugates. In many cases, the assignment of certain structural motifs such as fucosylation and sialylation is sufficient for diagnostics or batch monitoring. The occurrence of specific glycan epitopes is for example associated with several human diseases and a rapid diagnostic screening tool for these epitope features is therefore highly desired [36].

Recently, differences in sialic acid linkages were identified by analysing trisaccharide fragments derived from glycopeptide precursor ions (Figure $3 \mathrm{~b}$ ) $\left[37^{\bullet}, 3^{\circ}\right]$. The fragments derived from synthetic as well as biological glycopeptide precursor ions and exclusively contained either $a 2,3$ or $\alpha 2,6$ linked $\mathrm{N}$-acetylneuraminic acid (NeuAc) (Figure 3b, precursor structures). CID experiments of the glycopeptides resulted in the generation of diagnostic trisaccharide fragments, which showed characteristic drift times depending on the regiochemistry of the NeuAc moiety (Figure 3b, fragment spectra). The results of these studies show that a rapid identification and unambiguous assignment of glycan motifs can be performed independent of the nature and origin of the investigated sample. In addition, the method is fast and does not require derivatisation, therefore demonstrating the potential of IM-MS as a high-throughput method to screen larger glycans and glycoconjugates for specific biomarkers.

\section{Gas-Phase Spectroscopy}

Molecular spectroscopy provides a sensitive method for studying isolated molecules in the gas-phase under controlled conditions. In contrast to the overall size and shape information derived from IMS, gas-phase spectroscopy can deliver specific information, for example on the secondary structure of the investigated molecules [39]. Due to the ability to obtain details about functional groups, hydrogen bonding and preferred molecular conformations, gas-phase IR spectroscopy is well established for the structural analysis of peptides [39], proteins [40] and small molecules [41]. IR spectroscopy has a high potential for elucidating different conformational and structural features, however to date the method has only been sparsely used for glycan analysis.

\section{IR Spectroscopy of Glycans}

The first gas-phase IR spectroscopy experiments on glycans were performed using molecular beam experiments, in which gasphase ions were generated through vaporization. A prominent example is the analysis of glycopyranosides [42]. Protonated ions were created in a molecular beam through proton transfer from photo-ionized phenol complexes and detected through infrared multiple photon dissociation (IRMPD). In combination with structural predictions using DFT and ab initio calculations, the obtained spectra allowed the assignment of the preferred monosaccharide conformations. Nevertheless, the transfer of the sample into the gas phase by vaporization limits the utility of the method to small molecules.

\section{IRMPD Spectroscopy}

The application of electrospray ionization (ESI) to gently transfer analytes into the gas phase enabled the spectroscopic analysis of much larger analytes. In addition, the combination of MS/IR enables $\mathrm{m} / \mathrm{z}$-selection of ions prior to spectroscopy and therefore allows the individual analysis of distinct species even in complex samples [43]. IRMPD approaches typically employ IR radiation 
with high laser fluence. The sequential absorption of multiple photons usually provides sufficient energy to break the weakest bond. The resulting fragment can be detected by MS [44]. IR spectra are constructed by plotting the fragmentation yield as a function of laser frequency. However, IRMPD spectra of glycans at room temperature suffer from spectral congestion and low resolution even at the mono- [45] and disaccharide [46] level (Figure 4a, purple trace). There are two major phenomena, which lead to this spectral congestion: 1) The inherent conformational flexibility of carbohydrate ions in the gas phase, which appears to be quite high at room temperature and 2) the general mechanism of IRMPD. The absorption of multiple photons leads to considerable molecular heating of the ions before dissociation, resulting in hot/ activated molecules, which show broadened peaks in the IR spectrum [43]. Although spectral congestion complicates the application of IRMPD at room temperature, it is still of use for smaller systems such as monosaccharides [47 ${ }^{\circ}$. The monosaccharides L-iduronic acid and D-glucoronic acid for example, are major building blocks of glycosaminoglycans (GAGs). In a recent study, L-iduronic acid and D-glucoronic acid ions were generated from synthetic standards and analysed by IRMPD at $300 \mathrm{~K}$. The IR spectra show two major signals for both isoforms, which are very broad due to spectral congestion. However, the comparison of the spectra between the individual monosaccharides reveals shifts in the bands, which allows for their distinction. This enables the characterization of larger biopolymers using a fragmentbased approach similar to the fragment CCS analysis by IM-MS.

\section{Cold-Ion Spectroscopy}

Recently, the enormous potential of cold-ion gas-phase IR spectroscopy to elucidate glycan structures in great detail was demonstrated. A series of proof-of-principles experiments were performed using an isomeric set of six trisaccharides, which cannot be distinguished by LC-MS and were only recently characterised by IM-MS [20*,48*0. After transfer of the ions into the gas phase using nano ESI, they were $\mathrm{m} / \mathrm{z}$-selected and stored in a hexapole ion trap. Cooling of the ions to $\sim 0.4 \mathrm{~K}$ was achieved by encapsulation of the ions in superfluidic helium nanodroplets, which are transparent to IR radiation and interact only marginally with the trapped ion [40]. The doped droplets were then irradiated with IR photons from a tuneable free electron laser (FEL) [49], resulting in the wavelength-dependent resonant absorption of photons and subsequent ejection of the ions from the droplets. The resulting cold-ion IR spectra showed highly resolved bands leading to fingerprints that were highly diagnostic for each of the examined trisaccharides [48*0]. Comparison of the individual spectra showed that all possible types of isomerism can be distinguished using this technique, however, a detailed assignment of specific peaks on the basis of DFT calculations is difficult due to the flexibility of gas-phase glycan ions [50].

The above-mentioned He nanodroplet experiment represents the state of the art, but the instrumental setup is technically elaborate and the utilized FEL laser light is only accessible to few groups. More widely used instruments for cold-ion spectroscopy utilize messenger-tagging IR spectroscopy [51]. Tagging spectroscopy utilizes a weakly bound atom or molecule that is attached to the ion of interest. Absorption of one IR photon leads to the dissociation of this "messenger", therefore requiring lower laser fluences than in IRMPD [52]. Tagged complexes are usually interrogated by infrared laser pulses generated from tuneable benchtop OPA/OPO laser systems that are commercially available. Although tagging experiments [53] do not reach similar temperatures as in helium droplets experiments (<1 K) (Figure 4a, black trace) [40], the accessible temperature range was shown to be sufficiently low to obtain comparable, highly-resolved gas-phase IR spectra (Figure 4b) [52]. 

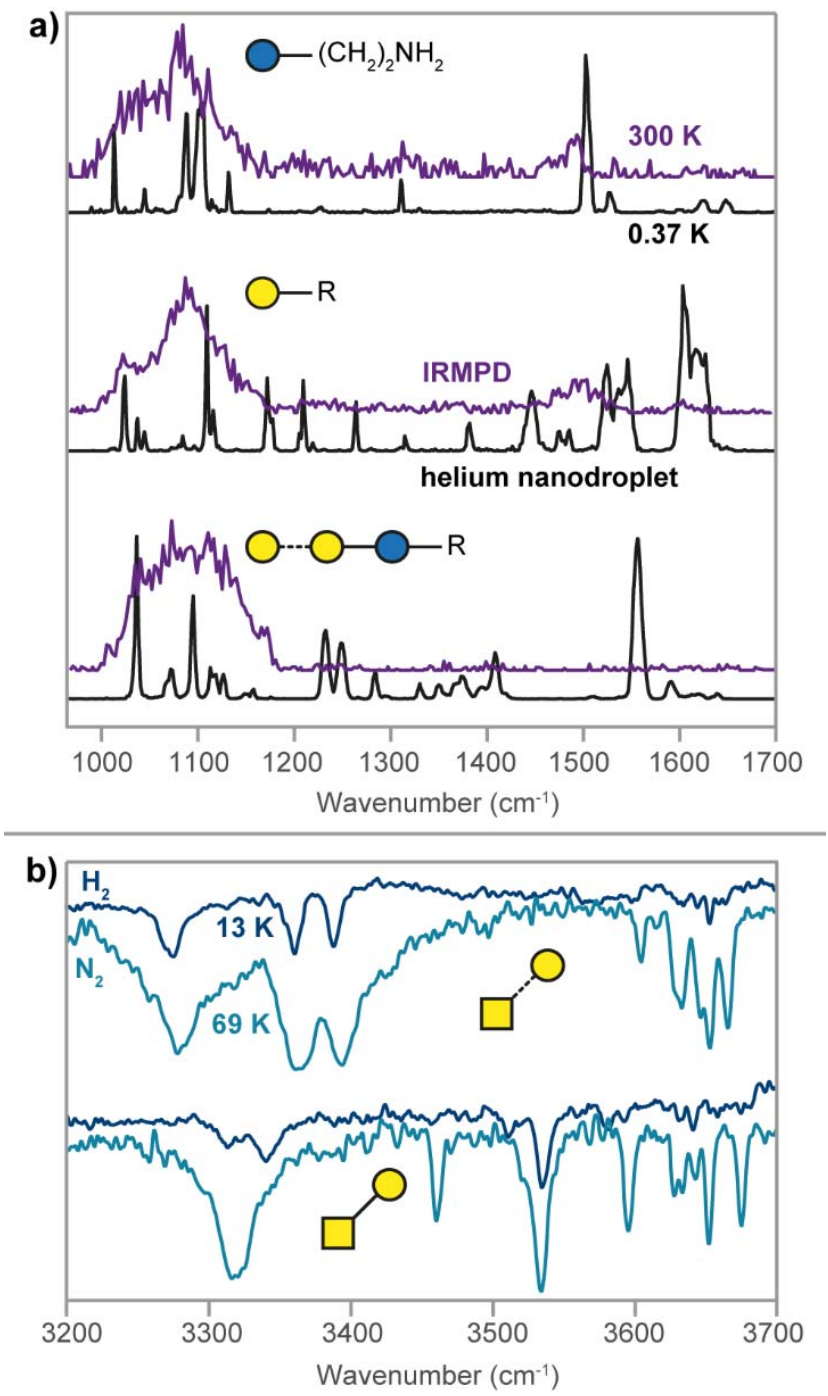

Figure 4: Comparison of different gas-phase IR spectroscopy techniques at different temperatures. a) Two isomeric monosaccharides and one trisaccharide measured with IRMPD at room temperature (purple trace) in comparison to helium nanodroplet experiments at $0.37 \mathrm{~K}$ (black trace) (Adapted by permission from Wiley-VCH Verlag GmbH \& Co. KGaA: Angewandte Chemie Int. Ed. [48*•], copyright 2017.). b) Tagging IR spectra of two isomeric disaccharides with either $\mathrm{H}_{2}$ at $13 \mathrm{~K}$ (blue trace) or $\mathrm{N}_{2}$ at $69 \mathrm{~K}$ (orange trace) as messengers (Adapted by permission from American Society for Mass Spectrometry: Journal of the American Society for Mass Spectrometry [54], copyright 2017).

In principle, a variety of suitable messenger molecules are available; often "innocent" rare gases (argon, neon, helium) or small molecules (hydrogen) are used to avoid perturbations in the spectrum caused by analyte-messenger interactions. First applications of tagging spectroscopy on glycans showed great promise. It was shown, that the particular challenging class of GAGs can be analysed using cryogenic messenger-tagging spectroscopy [55**]. Five GAG-based disaccharides, which merely differed in either their composition, connectivity, or sulfate group location were shown to yield very distinct cold-ion IR spectra using hydrogen as messenger tag.

The strength of room temperature IRMPD spectroscopy appears to lie in the analysis of smaller mono- and disaccharide fragments, cold-ion IR spectroscopy is able to unambiguously assign differences in larger, intact oligosaccharide precursor. Future experiments will show if the relatively straightforward and faster room temperature IR spectroscopy is also capable of characterizing larger oligosaccharide by applying top-down fragment strategies similar to IM-MS, or if more elaborate cold-ion spectroscopy techniques are required for an in-depth analysis. 


\section{Summary and Outlook}

In recent years, numerous experiments have illustrated the outstanding potential of IM-MS for the analysis of stereo- and regioisomers of complex glycans and glycoconjugates. It was demonstrated how mobility information can be used to reduce spectral complexity and how diagnostic features in glycan structures can be used for rapid identification. There are very few other methods that are able to provide a comparably extensive analysis of compositional, regio- as well as stereochemical information of glycans on such short time scales with similarly low sample consumption. With its universal separation capabilities, IM-MS has the potential to replace some, but certainly not all chromatographic methods in routine analysis.

However, IMS alone only provides information on the overall shape of the investigated ions; the separation power for extremely similar structural isomers such as compositional isomers is clearly limited. A promising approach to obtain more detailed information is the coupling of IM-MS to orthogonal techniques, most notably gas-phase IR spectroscopy. Cold-ion IR spectroscopy has highly promising diagnostic capabilities and can deliver information on structural details, which are difficult to obtain with other methods. Proof-of-principle experiments on a coupling between IM-MS and IR spectroscopy already showed great promise [54]; the utility for mixture analysis, however, remains to be shown. A suitable way to achieve such complex sample analysis would be for example to use IM-MS as pre-selection tool and coarse separation technique as demonstrated for group separation above. For the combination of IM-MS and IR spectroscopy into a user-friendly setup, major developments of software and database tools are furthermore required, and also a higher IM resolution would be desirable. Nevertheless, the combination of IM-MS-IR in conjunction with a database containing CCS as well as IR fingerprint reference data has the potential to serve as the major analytical tool for future glycan and glycoconjugate sequencing.

\section{Acknowledgments}

The authors thank Prof. David J. Harvey for fruitful discussions and continuous support.

\section{References and Recommended Reading}

Papers of particular interest, published within the period of review, have been highlighted as:

- of special interest

$\bullet$ of outstanding interest

1. Varki A: Biological roles of glycans. Glycobiology 2017, 27:3-49.

2. Hofmann J, Pagel K: Glycan Analysis by Ion Mobility-Mass Spectrometry. Angew Chem Int Ed Engl 2017, 10.1002/anie.201701309.

3. Varki A, Cummings RD, Aebi M, Packer NH, Seeberger PH, Esko JD, Stanley P, Hart G, Darvill A, Kinoshita T, et al.: Symbol Nomenclature for Graphical Representations of Glycans. Glycobiology 2015, 25:1323-1324.

4. Kanie O, Kanie Y: Addressing the glycan complexity by using mass spectrometry: In the pursuit of decoding glycologic. Biochemical Compounds 2017, 5:3.

5. Veillon L, Huang Y, Peng W, Dong X, Cho BG, Mechref $Y$ : Characterization of isomeric glycan structures by LC-MS/MS. Electrophoresis 2017, 10.1002/elps.201700042.

6. Kolarich D, Windwarder M, Alagesan K, Altmann F: Isomer-Specific Analysis of Released N-Glycans by LC-ESI MS/MS with Porous Graphitized Carbon. Methods Mol Biol 2015, 1321:427-435.

7. D'Atri V, Fekete S, Beck A, Lauber M, Guillarme D: Hydrophilic Interaction Chromatography Hyphenated with Mass Spectrometry: A Powerful Analytical Tool for the Comparison of Originator and Biosimilar Therapeutic Monoclonal Antibodies at the Middleup Level of Analysis. Anal Chem 2017, 89:2086-2092. 
8. Largy E, Cantais F, Van Vyncht G, Beck A, Delobel A: Orthogonal liquid chromatography-mass spectrometry methods for the comprehensive characterization of therapeutic glycoproteins, from released glycans to intact protein level. Journal of Chromatography A 2017, 1498:128-146.

9. Stavenhagen K, Plomp R, Wuhrer M: Site-Specific Protein N- and O-Glycosylation Analysis by a C18-Porous Graphitized CarbonLiquid Chromatography-Electrospray Ionization Mass Spectrometry Approach Using Pronase Treated Glycopeptides. Anal Chem 2015, 87:11691-11699.

10. Gaunitz S, Nagy G, Pohl NL, Novotny MV: Recent Advances in the Analysis of Complex Glycoproteins. Anal Chem 2017, 89:389413.

11. Lu H, Zhang Y, Yang P: Advancements in mass spectrometry-based glycoproteomics and glycomics. National Science Review 2016, 3:345-364.

12. Mantovani V, Galeotti F, Maccari F, Volpi N: Recent advances on separation and characterization of human milk oligosaccharides. Electrophoresis 2016, 37:1514-1524.

13. Ropartz D, Lemoine J, Giuliani A, Bittebiere Y, Enjalbert Q, Antoine R, Dugourd P, Ralet MC, Rogniaux H: Deciphering the structure of isomeric oligosaccharides in a complex mixture by tandem mass spectrometry: photon activation with vacuum ultraviolet brings unique information and enables definitive structure assignment. Anal Chim Acta 2014, 807:84-95.

14. May JC, McLean JA: Ion mobility-mass spectrometry: time-dispersive instrumentation. Anal Chem 2015, 87:1422-1436.

15. Li H, Bendiak B, Siems WF, Gang DR, Hill HH, Jr.: Ion Mobility-Mass Correlation Trend Line Separation of Glycoprotein Digests without Deglycosylation. Int J Ion Mobil Spectrom 2013, 16:105-115.

16. May JC, Goodwin CR, Lareau NM, Leaptrot KL, Morris CB, Kurulugama RT, Mordehai A, Klein C, Barry W, Darland E, et al.: Conformational Ordering of Biomolecules in the Gas Phase: Nitrogen Collision Cross Sections Measured on a Prototype High Resolution Drift Tube Ion Mobility-Mass Spectrometer. Anal Chem 2014, 86:2107-2116.

17. Pritchard LK, Harvey DJ, Bonomelli C, Crispin M, Doores KJ: Cell- and Protein-Directed Glycosylation of Native Cleaved HIV-1 Envelope. J Virol 2015, 89:8932-8944.

18. Harvey DJ, Sobott F, Crispin M, Wrobel A, Bonomelli C, Vasiljevic S, Scanlan CN, Scarff CA, Thalassinos K, Scrivens JH: Ion mobility mass spectrometry for extracting spectra of $\mathrm{N}$-glycans directly from incubation mixtures following glycan release: application to glycans from engineered glycoforms of intact, folded HIV gp120. J Am Soc Mass Spectrom 2011, 22:568-581.

19. Gray CJ, Thomas B, Upton R, Migas LG, Eyers CE, Barran PE, Flitsch SL: Applications of ion mobility mass spectrometry for high throughput, high resolution glycan analysis. Biochim Biophys Acta 2016, 1860:1688-1709.

20. Hofmann J, Hahm HS, Seeberger PH, Pagel K: Identification of carbohydrate anomers using ion mobility-mass spectrometry. Nature 2015, 526:241-244.

-• A study in which a set of six isomeric trisaccharides which differed in either composition, connectivity or configuration are compared. It systematically demonstrates the power of IM-MS to distinguish regio- and stereoisomeric glycans based on CCSs and $\mathrm{m} / \mathrm{z}$ information.

21. Harvey DJ, Scarff CA, Edgeworth M, Struwe WB, Pagel K, Thalassinos K, Crispin M, Scrivens J: Travelling-wave ion mobility and negative ion fragmentation of high-mannose N-glycans. J Mass Spectrom 2016, 51:219-235.

22. Campbell MP, Peterson R, Mariethoz J, Gasteiger E, Akune Y, Aoki-Kinoshita KF, Lisacek F, Packer NH: UniCarbKB: building a knowledge platform for glycoproteomics. Nucleic Acids Res 2014, 42:D215-221.

23. Struwe WB, Pagel K, Benesch JL, Harvey DJ, Campbell MP: GlycoMob: an ion mobility-mass spectrometry collision cross section database for glycomics. Glycoconj J 2016, 33:399-404.

24. Lisacek F, Mariethoz J, Alocci D, Rudd PM, Abrahams JL, Campbell MP, Packer NH, Stahle J, Widmalm G, Mullen E, et al.: Databases and Associated Tools for Glycomics and Glycoproteomics. Methods Mol Biol 2017, 1503:235-264. 
25. von Helden G, Hsu MT, Gotts N, Bowers MT: Carbon cluster cations with up to $\mathbf{8 4}$ atoms: structures, formation mechanism, and reactivity. The Journal of Physical Chemistry 1993, 97:8182-8192.

26. Mesleh MF, Hunter JM, Shvartsburg AA, Schatz GC, Jarrold MF: Structural Information from lon Mobility Measurements: Effects of the Long-Range Potential. The Journal of Physical Chemistry 1996, 100:16082-16086.

27. Shvartsburg AA, Jarrold MF: An exact hard-spheres scattering model for the mobilities of polyatomic ions. Chemical Physics Letters 1996, 261:86-91.

28. Deng L, Webb IK, Garimella SVB, Hamid AM, Zheng X, Norheim RV, Prost SA, Anderson GA, Sandoval JA, Baker ES, et al.: Serpentine Ultralong Path with Extended Routing (SUPER) High Resolution Traveling Wave lon Mobility-MS using Structures for Lossless Ion Manipulations. Anal Chem 2017, 89:4628-4634.

29. Huang $Y$, Dodds ED: Ion mobility studies of carbohydrates as group I adducts: isomer specific collisional cross section dependence on metal ion radius. Anal Chem 2013, 85:9728-9735.

30. Gaye MM, Nagy G, Clemmer DE, Pohl NL: Multidimensional Analysis of 16 Glucose Isomers by lon Mobility Spectrometry. Anal Chem 2016, 88:2335-2344.

31. Huang Y, Dodds ED: Discrimination of Isomeric Carbohydrates as the Electron Transfer Products of Group II Cation Adducts by Ion Mobility Spectrometry and Tandem Mass Spectrometry. Anal Chem 2015, 87:5664-5668.

-• A study in which carbohydrate/ metal ion interactions are used as means to improve discrimination of isomeric glycans. Suggests strategies for rapid differentiation of isomeric glycans by appropiate selection of metal ion adducts.

32. Li H, Bendiak B, Siems WF, Gang DR, Hill HH, Jr.: Ion mobility mass spectrometry analysis of isomeric disaccharide precursor, product and cluster ions. Rapid Commun Mass Spectrom 2013, 27:2699-2709.

33. Both P, Green AP, Gray CJ, Sardzik R, Voglmeir J, Fontana C, Austeri M, Rejzek M, Richardson D, Field RA, et al.: Discrimination of epimeric glycans and glycopeptides using IM-MS and its potential for carbohydrate sequencing. Nat Chem 2014, 6:65-74.

34. Hofmann J, Stuckmann A, Crispin M, Harvey DJ, Pagel K, Struwe WB: Identification of Lewis and Blood Group Carbohydrate Epitopes by Ion Mobility-Tandem-Mass Spectrometry Fingerprinting. Anal Chem 2017, 89:2318-2325

- A series of investigations on Lewis and blood group epitopes, which demonstrates the ability of IM-MS to monitor the fucosylation pattern and identify intact epitopes based on their fragment fingerprint.

35. Gaye MM, Kurulugama R, Clemmer DE: Investigating carbohydrate isomers by IMS-CID-IMS-MS: precursor and fragment ion cross-sections. Analyst 2015, 10.1039/c5an00840a.

36. Takada A, Ohmori K, Yoneda T, Tsuyuoka K, Hasegawa A, Kiso M, Kannagi R: Contribution of Carbohydrate Antigens Sialyl Lewis A and Sialyl Lewis X to Adhesion of Human Cancer Cells to Vascular Endothelium. Cancer Research 1993, 53:354-361.

37. Hinneburg H, Hofmann J, Struwe WB, Thader A, Altmann F, Varon Silva D, Seeberger PH, Pagel K, Kolarich D: Distinguishing Nacetylneuraminic acid linkage isomers on glycopeptides by ion mobility-mass spectrometry. Chem. Commun. 2016, 52:43814384 .

38. Guttman M, Lee KK: Site-Specific Mapping of Sialic Acid Linkage Isomers by Ion Mobility Spectrometry. Anal Chem 2016 , 88:5212-5217.

- Both papers simultaneously showed that the glycosylation of glycopeptides from different sources can be distinguished using IM-MS analysis of diagnostic fragment ions. It demonstrates the ability of IM-MS to serve as rapid screening technique when monitoring diagnostic features such as the sialylation pattern.

39. Seo J, Hoffmann W, Warnke S, Bowers MT, Pagel K, von Helden G: Retention of Native Protein Structures in the Absence of Solvent: A Coupled Ion Mobility and Spectroscopic Study. Angew. Chem. Int. Ed. Engl. 2016, 55:14173-14176.

40. Gonzalez Florez Al, Mucha E, Ahn DS, Gewinner S, Schollkopf W, Pagel K, von Helden G: Charge-Induced Unzipping of Isolated Proteins to a Defined Secondary Structure. Angew Chem Int Ed Engl 2016, 55:3295-3299.

41. Seo J, Warnke S, Gewinner S, Schollkopf W, Bowers MT, Pagel K, von Helden G: The impact of environment and resonance effects on the site of protonation of aminobenzoic acid derivatives. Phys Chem Chem Phys 2016, 18:25474-25482. 
42. Rudić S, Xie H-b, Gerber RB, Simons JP: Protonated sugars: vibrational spectroscopy and conformational structure of protonatedO-methyl $\alpha$-D-galactopyranoside. Molecular Physics 2012, 110:1609-1615.

43. Oomens J, Sartakov BG, Meijer G, von Helden G: Gas-phase infrared multiple photon dissociation spectroscopy of massselected molecular ions. International Journal of Mass Spectrometry 2006, 254:1-19.

44. Rijs AM, Oomens J: IR Spectroscopic Techniques to Study Isolated Biomolecules. Top Curr Chem 2015, 364:1-42.

45. Sagar R, Rudić S, Gamblin DP, Scanlan EM, Vaden TD, Odell B, Claridge TDW, Simons JP, Davis BG: Conformational effects in sugar ions: spectroscopic investigations in the gas phase and in solution. Chemical Science 2012, 3:2307.

46. Polfer NC, Valle JJ, Moore DT, Oomens J, Eyler JR, Bendiak B: Differentiation of isomers by wavelength-tunable infrared multiple-photon dissociation-mass spectrometry: application to glucose-containing disaccharides. Anal Chem 2006, 78:670679.

47. Schindler B, Renois-Predelus G, Bagdadi N, Melizi S, Barnes L, Chambert S, Allouche AR, Compagnon I: MS/IR, a new MS-based hyphenated method for analysis of hexuronic acid epimers in glycosaminoglycans. Glycoconj J 2017, 34:421-425.

- A combined MS/MS and room temperature IR study in which two isomeric monosaccharides were used to deduce the composition of a hyaluronic tetrasaccharide. Demonstrates the ability of IR to elucidate even complex glycans such as glycosaminoglycans.

48. Mucha E, Gonzalez Florez AI, Marianski M, Thomas DA, Hoffmann W, Struwe WB, Hahm HS, Gewinner S, Schollkopf W, Seeberger $\mathrm{PH}$, et al.: Glycan Fingerprinting via Cold-Ion Infrared Spectroscopy. Angew Chem Int Ed Engl 2017, 10.1002/anie.201702896.

-. Proof-of-principle study which demonstrates the power of cold-ion IR spectroscopy to obtain vibrational fingerprints of oligosaccharides. The well-resolved absorption features allow to distinguish between conncetivity, configuration, and even compositional isomers.

49. Schöllkopf W, Gewinner S, Junkes H, Paarmann A, von Helden G, Bluem H, Todd AMM: The new IR and THz FEL facility at the Fritz Haber Institute in Berlin. In SPIE Optics + OptoelectronicsEdited by Biedron SG: SPIE: 2015:95121L.

50. Marianski M, Supady A, Ingram T, Schneider M, Baldauf C: Assessing the Accuracy of Across-the-Scale Methods for Predicting Carbohydrate Conformational Energies for the Examples of Glucose and alpha-Maltose. J Chem Theory Comput 2016, 12:6157-6168.

51. Johnson CJ, Wolk AB, Fournier JA, Sullivan EN, Weddle GH, Johnson MA: Communication: He-tagged vibrational spectra of the SarGlyH(+) and $\mathrm{H}(+)(\mathrm{H}(2) \mathrm{O})(2,3)$ ions: quantifying tag effects in cryogenic ion vibrational predissociation (CIVP) spectroscopy. J Chem Phys 2014, 140:221101.

52. Altinay G, Metz RB: Comparison of IRMPD, Ar-tagging and IRLAPS for vibrational spectroscopy of Ag+(CH3OH). International Journal of Mass Spectrometry 2010, 297:41-45.

53. Bouchet A, Klyne J, Ishiuchi SI, Fujii M, Dopfer O: Conformation of protonated glutamic acid at room and cryogenic temperatures. Phys Chem Chem Phys 2017, 19:10767-10776.

54. Masellis C, Khanal N, Kamrath MZ, Clemmer DE, Rizzo TR: Cryogenic Vibrational Spectroscopy Provides Unique Fingerprints for Glycan Identification. J Am Soc Mass Spectrom 2017, 10.1007/s13361-017-1728-6.

55. Khanal N, Masellis C, Kamrath MZ, Clemmer DE, Rizzo TR: Glycosaminoglycan Analysis by Cryogenic Messenger-Tagging IR Spectroscopy Combined with IMS-MS. Anal Chem 2017, 89:7601-7606.

-. Demonstrates the potential of IM-MS together with cryogenic IR spectroscopy to unambiguously identify even complex glycans. The combined information of $\mathrm{m} / \mathrm{z}$, CCS, and IR fingerprint enables the differentiation of subtle structural differences of isomeric glycans. 